\title{
Long lasting hypercoagulability after subclinical COVID-19
}

\author{
Paolo Ferroli ${ }^{1}$ - Chiara Villa ${ }^{2}$ Andrea Ciuffi ${ }^{1} \cdot$ Guido Gubertini $^{3} \cdot$ Morgan Broggi $^{1}$ (I)
}

Published online: 5 August 2020

(c) Springer Science+Business Media, LLC, part of Springer Nature 2020

\section{Highlights}

- During COVID-19 pandemic emergency, a patient with a previous undiagnosed subclinical COVID-19 underwent urgent surgery for removal of a big intracranial tumor.

- The patient developed severe APE secondary to atrial catheter-related thrombosis in the post-craniotomy clinical course.

- Previous COVID-19 is suspected to be the main cause of the prothrombotic status of this patient, otherwise healthy and without any other risk factor for DVT/APE.

- The temporal evolution of the hypercoagulability state in COVID-19 is still unknown.

- Special attention is required when planning major surgical procedures in patients with previous COVID-19.

In the first months of 2020 Coronavirus disease 2019 (COVID-19) became pandemic; the clinical course of the disease and its potential complications still represent an open field of discussion. As pointed out by several authors, initial observations seem to indicate that a hypercoagulability state may be associated with COVID-19 and complicate the course of the disease [1-3]. We present and discuss the association between severe acute pulmonary embolism (APE) secondary to atrial catheter-related thrombosis in the post-craniotomy clinical course of a patient with a previous subclinical COVID-19. This case raises concerns about the duration of inflammatory state and hypercoagulability in this new disease.

Morgan Broggi

morgan.broggi@istituto-besta.it

1 Department of Neurosurgery, Fondazione IRCCS Istituto Neurologico Carlo Besta, Via Celoria, 11, 20133 Milano, Italy

2 Department of Radiology, Luigi Sacco Hospital, ASST Fatebenefratelli Sacco, Milano, Italy

3 Department of Infectious Diseases, Luigi Sacco Hospital, ASST Fatebenefratelli Sacco, Milano, Italy
On March 20th 2020, a severe APE secondary to thrombosis of a central venous catheter (Fig. 1a, b) was diagnosed post-operatively in a 39 years-old patient admitted to our ward, who was coming from a COVID-19 red zone (Bergamo district, north of Italy). Six weeks before admission he presented mild fever and cough that recovered spontaneously in few days; he did not further investigate these symptoms. Despite elective craniotomy restrictions due to the COVID19 emergency, he required urgent surgery because of a $4 \mathrm{~cm}$ suprasellar craniopharyngioma that was causing rapid decline of his visual function. The patient was otherwise healthy, without any comorbidity. Preoperative hematologic routine examinations did not disclose any abnormality. Complete trans-nasal endoscopic tumor resection was achieved without any immediate postoperative complications on March 16th 2020. His visual acuity rapidly improved and post-operative Magnetic Resonance confirmed complete resection of the lesion without any intracranial complications. Within postoperative medications (e.g. steroids, desmopressin), also standard prophylactic therapy for deep venous thrombosis (DVT) including intra and postoperative (until postoperative day (POD) 1, when the patient started walking) intermittent pneumatic compression and postoperative low molecular weight heparin (Enoxaparin, subcutaneous injection, $40 \mathrm{mg}$ daily) was administered to the patient.

However, on the afternoon of POD 2, the patient developed mild fever $\left(37.6^{\circ}\right)$ and cough. The upper respiratory tract swab real-time reverse transcription polymerase chain reaction (RT-PCR) for severe acute respiratory syndrome coronavirus 2 (SARS-COV-2) was negative. Chest X-Ray showed an irregular area of consolidation in the right lower lobe. Computed Tomography (CT) scan without i.v. contrast administration demonstrated many areas of "ground glass opacities" (GGO) distributed bilaterally and multiple gross sub-pleural fibrous stripes (Fig. 1c, d). GGO are commonly observed in the early stages of COVID-19 pneumonia $[4,5]$. Despite the negativity of the naso-pharyngeal swab, based on the patient's clinical and radiological features along with his region of origin, antiviral and antibiotic therapy was started. 

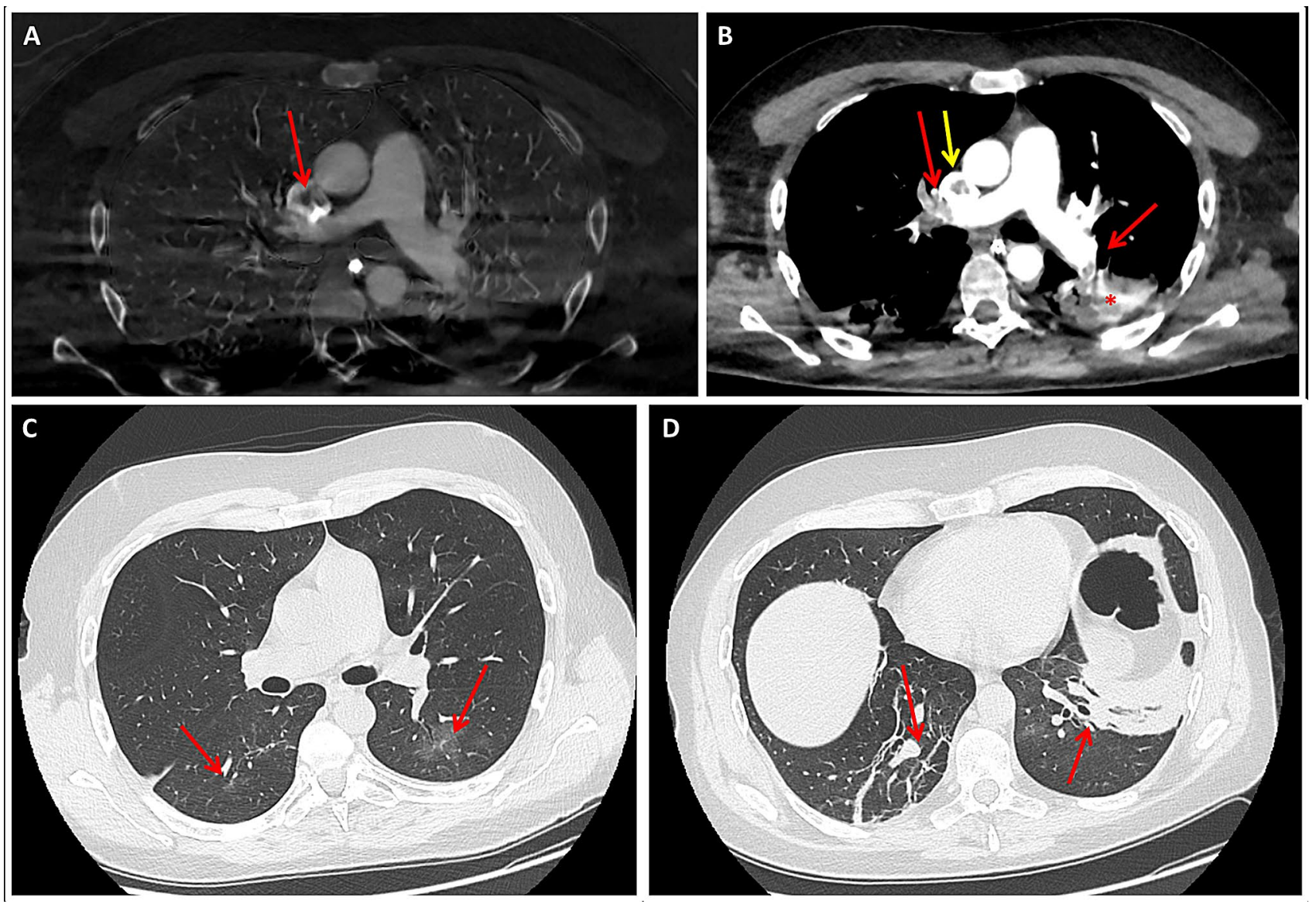

Fig. 1 a Chest CT with i.v. contrast administration showing a large central filling defect in the superior vena cava (thrombus) next to the tip of the central venous catheter (red arrow). b Chest CT with i.v. contrast administration depicting multiple filling defects within both pulmonary arteries involving lobar and segmental levels bilaterally, consistent with APE (red arrows). The posterior triangular subpleural

On POD 5, because of the persistence of fever (up to $38.3^{\circ}$ ) and cough and the development of tachycardia and dyspnea, the patient underwent a chest CT scan with i.v. contrast injection that disclosed multiple filling defects involving pulmonary arteries bilaterally at the lobar and segmental levels, consistent with APE originating from a central venous catheter related thrombus in his superior vena cava (Fig. 1a, b). CT scan also showed extensive consolidation areas involving the sub-pleural portions of the inferior lobes bilaterally and a small pleural effusion on the left; GGO were not visible any more. These findings were compatible with distal pulmonary infarctions complicating the APE; GGO, present on the first CT scan and considered as areas suspicious for initial alveolitis, were actually areas of mosaic oligaemia, that are also visible in initial phases of APE [6].

The patient rapidly deteriorated and required Intensive Care Unit (ICU) admission, intubation and mechanical respiratory assistance. Searches for SARS-COV-2 RNA in a consolidation visible in the lower left lobe is compatible with an area of infarction $(*)$. The caval thrombus is also visible (yellow arrow). c, d Chest CT without i.v. contrast administration showing ground glass opacities in the upper segments of both lower lobes (c, red arrows) and sub-pleural fibrous stripes in posterior regions (d, red arrows)

second nasal-pharyngeal swab and in two bronco-alveolar lavages resulted all negative. However, 12 days after patient admission, a test for SARS-COV-2 antibodies became available and the patient showed negative IgM, but positive IgG. We concluded that he had previous, undiagnosed, mild COVID-19 pneumonia, which might also explain the subpleural fibrotic scars visible on the first CT scan.

The patient was started on proper anticoagulant and support therapy; he progressively improved and on April 6th 2020 he was discharged from the ICU without any neurological and respiratory sequelae. In our tertiary referral neurosurgical department, this is the first case of severe central line-related APE in the last 20 years. Despite central venous catheters are well-known sources of thrombotic complications, severe APE has rarely been reported and it is generally associated with hypercoagulability states [7]. In our patient, however, the thrombophilia panel was within normal ranges and all common causes of a prothrombotic 
state were ruled out. On the other hand, brain tumors, like many other oncologic conditions, can be associated with a hypercoagulable state, but craniopharyngiomas have never been reported to produce this kind of condition. In light of the SARS-COV-2 antibodies test results, recent COVID-19 may be hypothesized as the main cause of this patient's prothrombotic status.

We acknowledge that the presented patient is just an association in a single case, but it does raise the idea that in patients with prior SARS-COV-2 infection, this might be considered an additional risk factor for DVT/APE warranting additional therapy and consideration to this issue.

It should be highlighted that are several conditions that mimic COVID-19 radiological features [4, 8], including other viral and bacterial infections, as well as cardiacinduced pulmonary edema and APE. As our case points out, in early stages of the diseases, COVID-19 and APE may share some common clinical and radiological features, like GGO like lesions.

The association between recent COVID-19 and this rare central line-related pulmonary complication should be a warning signal prompting more extensive studies on the duration of the hypercoagulability state during both the clinical course and convalescence of COVID-19. A clear relationship between the clinical progression of the disease and D-dimer and IL-6 plasmatic dosages has been reported in the acute phase [9], but we still do not have any idea of the temporal evolution of the inflammatory and coagulation state in the post-acute phase. Many concerns are raised by this case: which is the temporal path of the hypercoagulability state in COVID-19? Can this occur also in patients with a benign clinical course, as it happened in our case? How can we measure it? Do patients who need surgery after COVID19 require a special antithrombotic drug regimen? These, as many other questions, remain open issues in this difficult moment, while the medical community must learn how to deal with the multiple facets of this new disease.
Author contributions All authors contributed to the study conception and design, ant contributed equally to its development.

Funding All the authors are aware of the content of the paper, have read and approved it for submission to Journal of Thrombosis and Thrombolysis, and do not have any financial or other interests that might be construed as a conflict of interest. This work did not receive any specific grant from funding agencies in the public, commercial, or not-for-profit sectors.

\section{References}

1. Yin S, Huang M, Li D et al (2020) Difference of coagulation features between severe pneumonia induced by SARS-CoV2 and non-SARS-CoV2. J Thromb Thrombolysis. https://doi. org/10.1007/s11239-020-02105-8

2. Li T, Lu H, Zhang W (2020) Clinical observation and management of COVID-19 patients. Emerg Microbes Infect 9(1):687-690

3. Zhang Y, Cao W, Xiao M et al (2020) Clinical and coagulation characteristics of 7 patients with critical COVID-2019 pneumonia and acro-ischemia. Zhonghua Xue Ye Xue Za Zhi 41:E006

4. Ye Z, Zhang Y, Wang Y et al (2020) Chest CT manifestations of new coronavirus disease 2019 (COVID-19): a pictorial review. Eur Radiol. https://doi.org/10.1007/s00330-020-06801-0

5. Pan F, Ye T, Sun P et al (2020) Time course of lung changes on chest CT during recovery from 2019 Novel Coronavirus (COVID19) pneumonia. Radiology. https://doi.org/10.1148/radiol.20202 00370

6. Ruggiero A, Screaton NJ (2017) Imaging of acute and chronic thromboembolic disease: state of the art. Clin Radiol 72(5):375-388

7. Burns KE, McLaren A (2008) A critical review of thromboembolic complications associated with central venous catheters. Can J Anaesth 55(8):532-541

8. Dai W, Zhang H, Yu J et al (2020) CT imaging and differential diagnosis of COVID-19. Can Assoc Radiol J. https://doi. org/10.1177/0846537120913033

9. Gao Y, Li T, Han M et al (2020) Diagnostic utility of clinical laboratory data determinations for patients with severe COVID19. J Med Virol. https://doi.org/10.1002/jmv.25770

Publisher's Note Springer Nature remains neutral with regard to jurisdictional claims in published maps and institutional affiliations. 